\title{
A ratio-based method for ranking production units in profit efficiency measurement
}

\author{
Roza Azizi ${ }^{1} \cdot$ Reza Kazemi Matin ${ }^{1}$ Gholam R. Amin ${ }^{2}$
}

Received: 3 July 2016/Accepted: 16 October 2016/Published online: 8 November 2016

(C) The Author(s) 2016. This article is published with open access at Springerlink.com

\begin{abstract}
Evaluating profit efficiency measure needs input costs and output prices as well as input-output quantities. It is important to rank production units based on relative or absolute values of their production when costs and prices are available. Ranking production units in data envelopment analysis constitutes ranking individual units based on their profit efficiency ratio measures. Two novel models are presented in this paper for evaluating units based on a profit-dominance criterion. Models consider not only selfappraisal DEA optimal weights, but also all feasible input and output weights. A novel ratio-based and complete ranking method is introduced that is based on computing upper and lower boundaries for profit performance of observed units. An illustrative application of the models is then presented and results are discussed.
\end{abstract}

Keywords Data envelopment analysis - Profit efficiency · Ranking $\cdot$ Feasible weights

Reza Kazemi Matin

rkmatin@kiau.ac.ir

Roza Azizi

roza.azizi@kiau.ac.ir

Gholam R. Amin

gamin@squ.edu.om

1 Department of Mathematics, Karaj Branch, Islamic Azad University, Karaj, Iran

2 Department of Operations Management and Business Statistics, College of Economics and Political Science, Sultan Qaboos University, Muscat, Oman

\section{Introduction}

Evaluating performance of production systems and improving them as well as determining efficiency scores and inefficiency resources of production units are important issues for system managers. Data Envelopment Analysis, DEA, is a nonparametric optimization approach that was first introduced by Charnes et al. [1] to evaluate efficiency scores of decision-making units, DMUs. Basic DEA models are founded upon input-output data without the need for input costs and output prices. Some DEA models are available for cost, revenue, and profit analysis when costs and prices are available.

A profit efficiency model needs both input costs and output prices as discussed by Färe et al. [2, 3], while Färe and Grosskopf [4] derived cost and profit functions from directional technology distance function. More recent DEA efforts deal directly with profit efficiency from different perspectives. Examples include Chambers and Färe [5], Briec et al. [6], and Ruiz and Sirvent [7] among others.

Ranking is one of the more challenging items in performance management DEA that has received much attention. It is well known that the efficiency scores suggested by classical DEA models cannot fully discriminate efficient units, and inefficient units are evaluated by a mere set of optimal weights in a self-appraisal approach.

Sexton et al. [8] produced some of the first research that deals directly with the traditional ranking issues. Authors suggested a cross-efficiency matrix to rank units against self-appraisal weighting systems. However, cross-efficiency ranking method is inherently compromised by the existence of multiple optimal weights. To overcome this obstacle, Doyle and Green [9] suggested using weights as a secondary objective approach. The same approach is employed in some of the more recent efforts in this area, 
such as research conducted by Rodder and Reucher [10], Ruiz [11], or Yang et al. [12]. As an alternative approach, Anderson and Peterson [13] utilized a super-efficiency method for ranking units in DEA. To avoid over expenditure of efficiency for some units in Anderson and Peterson ranking method, Sueyoshi [14] suggested boundaries for weights. Seiford and Zhu [15] presented conditions that would render the super-efficiency method infeasible. Modified versions of super-efficiency method in ranking have been produced, such as those by Chen [16], Lee et al. [17], and Lee and Zhu [18]. A more recent ranking technique was introduced by Salo and Punkka [19], which considers all feasible weights and evaluates efficiency dominance relationships for each pair of DMUs by introducing ranking intervals.

There is much research devoted to profit efficiency and ranking methods in DEA literature. However, there is no direct approach for ranking units using profit efficiency scores. Common ranking methods, such as super-efficiency, do not consider input and output prices of DMUs and ignore vital information about them. Therefore, common methods are not accurate enough for ranking DMUs in the presence of prices.

The presented research introduces a new approach in ranking units by expanding upon the approach taken by Salo and Punkka [19] for profit models. All feasible weights of DMUs are calculated and units are ranked based upon their profit efficiency scores by taking the worst and the best profit rankings of a DMU in comparison with other DMUs. This paper will discuss the following:

- A novel approach to determining the best and the worst rankings of systems based on their profit efficiency.

- Models that consider all feasible weights and not only self- appraisal optimal DEA weights.

- Models providing ranking intervals for all systems and not only efficient ones.

- A numerical example demonstrating the applicability and efficiency of proposed models for hospitals.

Basic notations and workings of a DEA profit efficiency model are introduced in "Basic concept: illustration". "Ranking units based on profit efficiency score" will introduce ranking intervals and a novel ranking method based on profit efficiency scores. "Illustrative application" will demonstrate the new method through an illustrative example, and conclusions will be discussed in "Conclusions".

\section{Basic concept: illustration}

A two-dimensional example is used to illustrate the best and the worst possible ranking positions obtained from efficiency ratios. Let us start with the numerical example in
Table 1 Numerical example

\begin{tabular}{llll}
\hline DMU & Output 1 & Output 2 & Input \\
\hline A & 2 & 8 & 1 \\
B & 5 & 7 & 1 \\
C & 6 & 5 & 1 \\
D & 7 & 3 & 1 \\
E & 2.5 & 3.5 & 1 \\
F & 5 & 3 & 1 \\
\hline
\end{tabular}

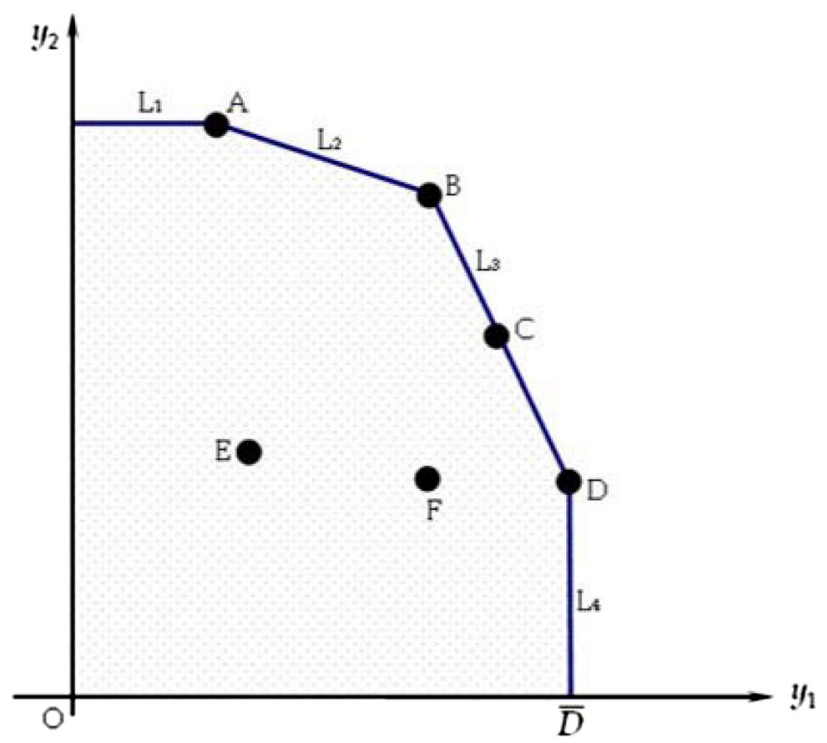

Fig. 1 PPS for numerical example in Table 1

Table 2 Best and worst rankings

\begin{tabular}{lll}
\hline DMUs & $r_{k}^{\min }$ & $r_{k}^{\max }$ \\
\hline A & 1 & 6 \\
B & 1 & 4 \\
C & 1 & 3 \\
D & 1 & 6 \\
E & 3 & 6 \\
F & 2 & 6 \\
\hline
\end{tabular}

Table 1. Example consists of six DMUs with two outputs and a single identical input as indicated.

Figure 1 presents the Production Possibility Set, PPS, for the example in Table 1.

There are four efficient DMUs A, B, C, D and two inefficient ones $\mathrm{E}$ and $\mathrm{F}$ shown in Fig. 1.

Data in Table 2 are produced using models proposed by Salo and Punkka [19] for determining the best and the worst ranking positions of DMUs.

The best and the worst ranking positions are graphically perceived by first considering the efficient frontier and its 
Table 3 Efficiency scores of six DMUs

\begin{tabular}{lllll}
\hline DMUs & $L_{1}$ & $L_{2}$ & $L_{3}$ & $L_{4}$ \\
\hline A & 1.000 & 1.000 & 0.706 & 0.286 \\
B & 0.875 & 1.000 & 1.000 & 0.714 \\
C & 0.625 & 0.808 & 1.000 & 0.857 \\
D & 0.375 & 0.615 & 1.000 & 1.000 \\
E & 0.438 & 0.500 & 0.500 & 0.357 \\
F & 0.375 & 0.538 & 0.765 & 0.714 \\
\hline
\end{tabular}

corresponding supporting hyperplanes. Four different supporting hyperplanes $L_{1}$ through $L_{4}$ may be denoted in Fig. 1 . Each supporting hyperplane is equivalent to an optimal solution of a CCR DEA model corresponding to at least one DMU. $L_{1}$ and $L_{2}$ are obtained from alternative optimal solutions of DEA model corresponding to DMU-A, while $L_{2}$ and $L_{3}$ are obtained from alternative optimal solutions of DMU-B and so on.

Mathematical equations corresponding to the supporting hyperplanes are as follows:

$L_{1}: 0.125 y_{2}=1$

$L_{2}: 0.038462 y_{1}+0.115385 y_{2}=1$

$L_{3}: 0.117647 y_{1}+0.058824 y_{2}=1$

$L_{4}: 0.142857 y_{1}=1$.

Each supporting hyperplane supplies an efficiency score to the six DMUs, and Table 3 summarizes these scores.

Table 3 may be used to interpret the best and the worst ranking positions for each DMU given in Table 2. The best ranking for DMU-A would be equal to 1 which is clearly obtained from the two passing supporting hyperplanes $L_{1}$ and $L_{2}$. In addition, the worst ranking position for DMU-A is equal to 6 which is obtained from the supporting hyperplane $L_{4}$ where the corresponding efficiency score of DMU-A given in the last column of Table 3, or 0.286 is the lowest score among the six DMUs. Other values in Table 2 may be interpreted similarly. It must be noted that some of the ranks of the intervals in Table 2 may not be obtained from Table 3 , because they are calculated by considering all feasible weights and not only the optimal ones.

The concept of utilizing profit efficiency determination as the bare model in determining the best and the worst ranking positions is discussed in the next section.

\section{Ranking units based on profit efficiency score}

It is assumed throughout this write up that there are $n$ DMUs which consume $m$ inputs to produce $s$ outputs. In addition, input and output vectors are denoted by $\boldsymbol{x}_{1}=$ $\left(x_{11}, \ldots, x_{\mathrm{ml}}\right)$ and $\boldsymbol{y}_{l}=\left(y_{11}, \ldots, y_{\mathrm{sl}}\right)$.
Efficiency of any $\mathrm{DMU}_{\mathrm{k}}$ under CRS assumption and in the absence of prices is the optimal value of the following CCR model:

$$
\begin{aligned}
& \beta^{*}=\min _{\boldsymbol{u}, \boldsymbol{v}} \boldsymbol{u}^{\prime} \boldsymbol{y}_{k} \\
& \boldsymbol{v}^{\prime} \boldsymbol{x}_{k}=1 \\
& \text { s.t. } \quad \boldsymbol{u}^{\prime} \boldsymbol{y}_{l}-\boldsymbol{v}^{\prime} \boldsymbol{x}_{l} \leq 0 \quad \text { for } l=1, \ldots, n \\
& \boldsymbol{u} \geq \boldsymbol{0}, \boldsymbol{v} \geq \boldsymbol{0}
\end{aligned}
$$

where $u=\left(u_{1}, \ldots, u_{\mathrm{s}}\right)$ and $v=\left(v_{1}, \ldots, v_{\mathrm{m}}\right)$ are the weight vectors of inputs and outputs. Weight vectors are assumed to be nonnegative.

Optimal weights in the above model vary across units, but preferred information on relative values of inputs and outputs is captured by their respective weights. When input and output prices are available, evaluating that profit efficiency is contained in producing an output vector $\boldsymbol{y}$ using an input vector $\boldsymbol{x}$ at maximum profit.

Assuming, input price vector for all units is equal to $c$ and output price vector for all units is equal to $\boldsymbol{p}$, actual profit for $\mathrm{DMU}_{k}$ would then be equal to $\boldsymbol{p} \boldsymbol{y}_{\boldsymbol{k}}-\boldsymbol{c} \boldsymbol{x}_{\boldsymbol{k}}$ and the maximum profit of $\boldsymbol{y}_{k}$ production by $\boldsymbol{x}_{k}$ consumption may be calculated by the following model as presented by Färe et al. [2, 3]:

$$
\begin{aligned}
& \beta^{*}=\max _{\boldsymbol{x} \boldsymbol{y}, \boldsymbol{\lambda}} \frac{\boldsymbol{p}^{\prime} \boldsymbol{y}-\boldsymbol{c}^{\prime} \boldsymbol{x}}{\boldsymbol{p}^{\prime} \boldsymbol{y}_{k}-\boldsymbol{c}^{\prime} \boldsymbol{x}_{k}} \\
& \text { s.t. } \quad \boldsymbol{x} \geq \sum_{\boldsymbol{j}} \boldsymbol{x}_{j} \lambda_{j} \\
& \boldsymbol{y} \leq \sum_{\boldsymbol{j}} \boldsymbol{y}_{\boldsymbol{j}} \lambda_{j} \\
& \boldsymbol{\lambda} \geq \boldsymbol{0} .
\end{aligned}
$$

To avoid creating unbounded solutions, Cooper et al. [20] modified the profit model as the following:

$\beta_{k}^{*}=\frac{\boldsymbol{p}^{\prime} \boldsymbol{y}^{*}-\boldsymbol{c}^{\prime} \boldsymbol{x}^{*}}{\boldsymbol{p}^{\prime} \boldsymbol{y}_{k}-\boldsymbol{c}^{\prime} \boldsymbol{x}_{k}}=\max _{\boldsymbol{x}, \boldsymbol{y}, \lambda} \frac{\boldsymbol{p}^{\prime} \boldsymbol{y}-\boldsymbol{c}^{\prime} \boldsymbol{x}}{\boldsymbol{p}^{\prime} \boldsymbol{y}_{k}-\boldsymbol{c}^{\prime} \boldsymbol{x}_{k}}$

s.t. $\quad \boldsymbol{x}=\sum_{j} \boldsymbol{x}_{j} \lambda_{j} \leq \boldsymbol{x}_{k}$

$\boldsymbol{y}=\sum_{j} y_{j} \lambda_{j} \geq y_{k}$

$\lambda \geq \boldsymbol{0}$.

Profit efficiency score for $\mathrm{DMU}_{k}$ is then measured as $\mathrm{PE}_{k}=\frac{1}{\beta_{k}^{*}}=\frac{\boldsymbol{p}^{\prime} \boldsymbol{y}_{k}-\boldsymbol{c}^{\prime} \boldsymbol{x}_{k}}{\boldsymbol{p}^{\prime} \boldsymbol{y}^{*}-\boldsymbol{c}^{\prime} \boldsymbol{x}^{*}}$. Therefore, $0<\mathrm{PE}_{k} \leq 1$ under the assumption of $\boldsymbol{p}^{\prime} \boldsymbol{y}_{k}>\boldsymbol{c}^{\prime} \boldsymbol{x}_{k}$. $\mathrm{DMU}_{k}$ is profit efficient if and only if $\mathrm{PE}_{k}=1$.

The dual formula in Model 3 may be presented by the following Model 4: 


$$
\begin{aligned}
& \beta_{k}^{*}=\min _{\boldsymbol{u}, \boldsymbol{v}}-\boldsymbol{u}^{\prime} \boldsymbol{y}_{k}+\boldsymbol{v}^{\prime} \boldsymbol{x}_{k} \\
& \text { s.t. } \quad \boldsymbol{u}^{\prime} \boldsymbol{y}_{l}-\boldsymbol{v}^{\prime} \boldsymbol{x}_{l}+\overline{\boldsymbol{p}}^{\prime} \boldsymbol{y}_{l}-\overline{\boldsymbol{c}}^{\prime} \boldsymbol{x}_{l} \leq 0 \quad \text { for } l=1, \ldots, n \\
& \overline{\boldsymbol{c}}=\frac{\boldsymbol{c}}{\boldsymbol{p}^{\prime} \boldsymbol{y}_{k}-\boldsymbol{c}^{\prime} \boldsymbol{x}_{k}} \\
& \overline{\boldsymbol{p}}=\frac{\boldsymbol{p}}{\boldsymbol{p}^{\prime} \boldsymbol{y}_{k}-\boldsymbol{c}^{\prime} \boldsymbol{x}_{k}} \\
& \boldsymbol{u} \geq \boldsymbol{0}, \boldsymbol{v} \geq \boldsymbol{0} .
\end{aligned}
$$

Note that $\bar{c}$ and $\bar{p}$ are input cost and output price vectors, respectively. Values are normalized by the observed profit of the unit under evaluation.

Using the second and third equations in Model 4, $\overline{\boldsymbol{p}}^{\prime} \boldsymbol{y}_{k}-\overline{\boldsymbol{c}}^{\prime} \boldsymbol{x}_{k}=1$ is obtained. Therefore, model may be rewritten as:

$$
\begin{aligned}
& \beta_{k}^{*}=\min _{\boldsymbol{u}, \boldsymbol{v}} \frac{-\boldsymbol{u}^{\prime} \boldsymbol{y}_{k}+\boldsymbol{v}^{\prime} \boldsymbol{x}_{k}}{\overline{\boldsymbol{p}}^{\prime} \boldsymbol{y}_{k}-\overline{\boldsymbol{c}}^{\prime} \boldsymbol{x}_{k}} \\
& \text { s.t. } \quad \boldsymbol{u}^{\prime} \boldsymbol{y}_{l}-\boldsymbol{v}^{\prime} \boldsymbol{x}_{l}+\overline{\boldsymbol{p}}^{\prime} \boldsymbol{y}_{l}-\overline{\boldsymbol{c}}^{\prime} \boldsymbol{x}_{l} \leq 0 \quad \text { for } l=1, \ldots, n \\
& \overline{\boldsymbol{c}}=\frac{\boldsymbol{c}}{\boldsymbol{p}^{\prime} \boldsymbol{y}_{k}-\boldsymbol{c}^{\prime} \boldsymbol{x}_{k}} \\
& \overline{\boldsymbol{p}}=\frac{\boldsymbol{p}}{\boldsymbol{p}^{\prime} \boldsymbol{y}_{k}-\boldsymbol{c}^{\prime} \boldsymbol{x}_{k}} \\
& \boldsymbol{u} \geq \boldsymbol{0}, \boldsymbol{v} \geq \boldsymbol{0} .
\end{aligned}
$$

Profit efficiency score of each DMU is dependent on its corresponding optimal output and input weights, or shadow prices derived from the above linear programming Model 5. It must be noted that optimal output and input weights vary across firms under study.

More general constraints on the relative importance of output and input weights may be imposed to avoid zero weights and to apply decision maker preferred information to outputs and inputs. Examples include choosing input and output weights from restricted sets $U \subseteq \mathbb{R}_{++}^{s}$ and $V \subseteq \mathbb{R}_{++}^{m}$ derived in the Cone-Ratio approach by Charnes et al. [21] or in Assurance Region approach introduced by Thompson et al. [22].

Best and worst profit rankings are determined in each DMU by considering possible choices of DEA weights as a collective consequence of all DMUs. The workings of the presented method are first demonstrated by an example using data from Table 1 . Weight restrictions in Table 1 are taken as $\boldsymbol{u}, \boldsymbol{v} \geq \boldsymbol{0}$. In addition, input vector for all units $(\boldsymbol{c})$ is set as 2 and output price vector for all units $(\boldsymbol{p})$ is taken as equal to 5 .

Using arbitrary feasible weights, Table 4 is obtained for efficiency scores of 6 DMUs.

First column in Table 4 indicates the profit efficiency score of each unit based on optimal weights for DMU-C and its respective $\overline{\boldsymbol{p}}$ and $\overline{\boldsymbol{c}}$. Next, three columns indicate the profit efficiency scores of each unit based on three sets of arbitrary feasible weights besides $\bar{p}$ and $\bar{c}$. of DMU-C. $u_{i}$
Table 4 Profit efficiency score of 6 DMUs based on arbitrary feasible weights

\begin{tabular}{lllll}
\hline DMUs & $C$ & $\begin{array}{l}u_{1}=0.0 \\
u_{2}=0.1 \\
v=1.9\end{array}$ & $\begin{array}{l}u_{1}=0.3 \\
u_{2}=0.0\end{array}$ & $\begin{array}{l}u_{1}=0.25 \\
u_{2}=0.1 \\
v=3.2\end{array}$ \\
\hline A & 0.638 & 0.976 & 0.447 & 0.565 \\
B & 1.000 & 0.976 & 0.780 & 0.937 \\
C & 1.000 & 0.714 & 0.833 & 0.833 \\
D & 1.000 & 0.518 & 0.921 & 0.721 \\
E & 0.351 & 0.362 & 0.249 & 0.252 \\
F & 0.583 & 0.427 & 0.455 & 0.414 \\
\hline
\end{tabular}

$(i=1,2)$ and $v$ represent the arbitrary feasible weights of their corresponding outputs and inputs, respectively.

It may be ascertained now that worst profit ranking of any DMU signifies the number of DMUs which have at least as high of profit efficiency scores as the one under evaluation. Moreover, best ranking indicates the number of DMUs that have a higher efficiency score than the one under evaluation.

As evident in Table 4, DMU-C has the highest profit efficiency score of all DMUs. Therefore, DMU-C may have a best ranking of 1 and a value of 3 at its worst ranking, because the other two DMUs have as high of profit efficiency scores as DMU-C. Similarly, the best and the worst rankings of DMU-C in the other three columns are 3, 2, and 2.

Since analyzing all feasible weights is not possible, a new approach of evaluating all feasible weights and not just optimal weights must be considered to rank DMUs based on their profit efficiency scores. However, considering this approach necessitates introduction of new sets and notations. Therefore, certain sets and notations are introduced to present the approach proposed in this write up.

The following sets determine the indexes of DMUs with strictly higher profit efficiency scores than $\mathrm{DMU}_{k}\left(\mathrm{PR}_{k<}\right)$ or at least as high of profit efficiency score under a common set of output-input weights. Sets are defined as per the following:

$$
\begin{aligned}
\mathrm{PR}_{k<} & =\left\{l \in\{1, \ldots, \mathrm{n}\} \mid P E_{l}(\boldsymbol{u}, \boldsymbol{v})>P E_{k}(\boldsymbol{u}, \boldsymbol{v})\right\} \\
& =\left\{l \in\{1, \ldots, \mathrm{n}\} \mid \beta_{l}^{*}(\boldsymbol{u}, \boldsymbol{v})<\beta_{k}^{*}(\boldsymbol{u}, \boldsymbol{v})\right\} \\
\mathrm{PR}_{k} \leq & =\left\{l \in\{1, \ldots, \mathrm{n}\}-\{\mathrm{k}\} \mid P E_{l}(\boldsymbol{u}, \boldsymbol{v}) \geq P E_{k}(\boldsymbol{u}, \boldsymbol{v})\right\} \\
& =\left\{l \in\{1, \ldots, \mathrm{n}\}-\{\mathrm{k}\} \mid \beta_{l}^{*}(\boldsymbol{u}, \boldsymbol{v}) \leq \beta_{k}^{*}(\boldsymbol{u}, \boldsymbol{v})\right\} .
\end{aligned}
$$

Corresponding profit efficiency ranking may then be defined as follows: $\operatorname{pr}_{k<}=1+\left|\mathrm{PR}_{k<}\right|$ and $\mathrm{pr}_{k \leq}=$ $1+\left|\mathrm{PR}_{k} \leq\right|$ in the above sets, where $\|$ shows cardinality number of the set.

Based on the above relations, there exist feasible weights for $\mathrm{DMU}_{\mathrm{k}}$ that make the unit profit efficient. That is to say, if there is no DMUs with strictly higher profit 
efficiency score than $\mathrm{DMU}_{k}$, then $\mathrm{pr}_{k<}=1$, and $\mathrm{pr}_{k \leq}$ is equal to the number of all profit efficient DMUs plus one. Then again, should $\mathrm{DMU}_{\mathrm{k}}$ be profit inefficient for some feasible weights, then $\mathrm{pr}_{k<}$ and $\mathrm{pr}_{k \leq}$ would increase according to the number of DMUs that have higher profit efficiency scores than $\mathrm{DMU}_{\mathrm{k}}$ or at least have the same profit efficiency scores as $\mathrm{DMU}_{\mathrm{k}}$. When utilizing all feasible weights, it is sufficient to minimize $\mathrm{pr}_{k<}$ and maximize $\mathrm{pr}_{k} \leq$ over the feasible weight spectrum to determine the best and the worst profit rankings of $\mathrm{DMU}_{k}$.

Salo and Punkka [19] method may now be extended to present the following models for determining profit efficiency ranking intervals.

$$
\begin{aligned}
& \min _{z, \boldsymbol{u}, \boldsymbol{v}} 1+\sum_{l \neq k} z_{l} \\
& \text { s.t. } \quad-\boldsymbol{u}^{\prime} \boldsymbol{y}_{k}+\boldsymbol{v}^{\prime} \boldsymbol{x}_{k}=1 \\
& \boldsymbol{u}^{\prime} \boldsymbol{y}_{l}-\boldsymbol{v}^{\prime} \boldsymbol{x}_{l}+\overline{\boldsymbol{p}}^{\prime} \boldsymbol{y}_{l}-\overline{\boldsymbol{c}}^{\prime} \boldsymbol{x}_{l} \\
& \leq M_{k} z_{l} l \neq k \\
& \boldsymbol{z}_{l} \in\{0,1\} \quad \text { for } l \neq k \\
& \boldsymbol{u} \in U, \boldsymbol{v} \in V \\
& \max _{z, \boldsymbol{u}, \boldsymbol{v}} 1+\sum_{l \neq k} z_{l} \\
& \text { s.t. } \quad-\boldsymbol{u}^{\prime} \boldsymbol{y}_{k}+\boldsymbol{v}^{\prime} \boldsymbol{x}_{k}=1 \\
& -\boldsymbol{u}^{\prime} \boldsymbol{y}_{l}+\boldsymbol{v}^{\prime} \boldsymbol{x}_{l}-\overline{\boldsymbol{p}}^{\prime} \boldsymbol{y}_{l}+\overline{\boldsymbol{c}}^{\prime} \boldsymbol{x}_{l} \\
& \leq M_{k}\left(1-z_{l}\right) l \neq k \\
& \boldsymbol{z}_{l} \in\{0,1\} \quad \text { for } l \neq k \\
& \boldsymbol{u} \in U, \boldsymbol{v} \in V .
\end{aligned}
$$

$M_{k}$ is the smallest positive constant in the above that makes the models feasible for unit $k$. However, $M_{k}$ may not be identical in both models.

Models 6 and 7 are used in determining the minimum number of DMUs that have higher efficiency scores than $\mathrm{DMU}_{k}$. The same models are also used in determining the maximum number of DMUs that have at least as high of efficiency scores as $\mathrm{DMU}_{k}$. Similar methodology may be utilized to illustrate ranking intervals of DMUs, or to determine the best and worst profit efficiency rankings of DMUs in Variable Return to Scale, VRS technology.

Ranking intervals in profit efficiency evaluation of observed units may be obtained by utilization of the following Theorems 1 and 2:

Theorem 1 Optimal objective value in Model 6 is the best profit ranking of $D M U_{k}$.

Theorem 2 Optimal objective value in Model 7 is the worst profit ranking of $D M U_{k}$.
Proofs for Theorems 1 and 2 are presented in "Appendix".

\section{Illustrative application}

Assume a set of data for 14 hospitals having two inputs and two outputs each. Inputs are number of doctors and nurses and outputs are number of outpatient and inpatient visits. This is the same input-output data as used in Cooper et al. [20].

Models 6 and 7 are utilized with weight restrictions of $\boldsymbol{u}, \boldsymbol{v} \geq \boldsymbol{0}$. Common input prices are taken as 5 and 2, and common output prices are chosen as 3 and 7 , respectively. Table 5 illustrates profit efficiency scores of the hospitals in column 2, and the best and the worst profit efficiency rankings in columns 3 and 4, respectively.

Profit efficient Hospitals 2, 3, 6, 8, and 10 achieve the best possible performance for certain feasible weights, and their profit ranking intervals of are [1,7], [1,2], [1,5], $[1,11]$, and $[1,1]$, respectively. This demonstrates that their rankings are flexible within their corresponding intervals according to different feasible weights. Units are unable to have the worst ranking of 14 among feasible input-output weights.

Hospital 10 in its worst possible scenario performs better than all other units for certain feasible weights; therefore, it may be stated that this unit has the best performance among all units. Meanwhile, Hospital 8 in the worst possible case performs better than only three other units for certain feasible weights; therefore, this unit would have the worst performance among all units.

Table 5 Profit efficiency scores, and the best and the worst rankings of 14 hospitals

\begin{tabular}{llcc}
\hline Hospital & Profit efficiency & Best rank & Worst rank \\
\hline 1 & 0.858 & 7 & 9 \\
2 & 1 & 1 & 7 \\
3 & 1 & 1 & 2 \\
4 & 0.597 & 11 & 14 \\
5 & 0.814 & 8 & 11 \\
6 & 1 & 1 & 5 \\
7 & 0.772 & 10 & 12 \\
8 & 1 & 1 & 11 \\
9 & 0.947 & 2 & 3 \\
10 & 1 & 1 & 1 \\
11 & 0.872 & 6 & 10 \\
12 & 0.909 & 4 & 9 \\
13 & 0.691 & 12 & 14 \\
14 & 0.912 & 3 & 14 \\
\hline
\end{tabular}


Consider three Hospitals 4, 13, and 14. As evident from Table 5, these hospitals may obtain the worst possible rank 14 with nonnegative input and output weights. Hospital 13 has the worst performance among the three hospitals, because certain feasible weights would lead this unit to be ranked 12 at its best ranking. This indicates that hospital unit 13 may be better than only two other hospital units at best.

Figure 2 is a column chart for efficiency rankings of the hospital units that visually illustrate the results.

Individual columns in Fig. 2 refer to the 14 hospital units under study and indicate their individual profit efficiency ranking intervals. Horizontal axis in the chart indicates the hospital unit, and the vertical axis is a measure of their respective profit efficiency ranking. The bottom of each column indicates the best profit efficiency ranking for the hospital unit, while the top of the column indicates its worst ranking.

The height of each column displays the profit efficiency ranking that could be achieved by each hospital based on different feasible weights. Note that the produced rankings are just natural numbers. Profit efficiency ranking of hospital 1 could not be less than seven or more than nine. Hospitals 8 and 14 exhibit the largest range of variations in their profit rankings, and the smallest variations are displayed in Hospitals 3, 9, and 10. Although both Hospitals 3 and 9 could be ranked on two levels for all feasible weights, performance of Hospital 3 is better than that of Hospital 9, because the performances of its best and worst profit rankings are better.

Interval range of some hospitals, such as Hospitals 8 and 14 , is larger than others due to inclusion of all their feasible weights and not only their self-appraisal DEA optimal weights. To illustrate this point, ranking interval for hospital 8 is calculated to be $[1,11]$. This means that its ranking varies between 1 as the best and 11 as the worst depending on the set of feasible weights. For example, there is at least one set of feasible weight that allows this hospital to rank as the 7th best performing hospital.

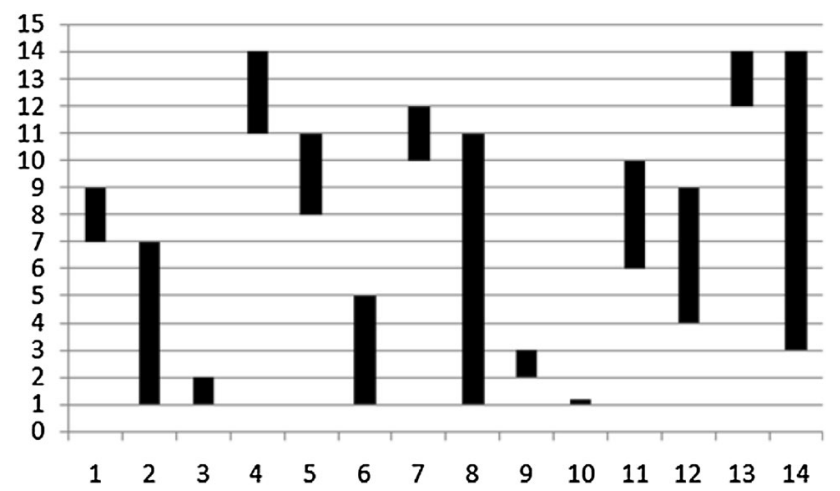

Fig. 2 Profit efficiency ranking interval of 14 hospitals
Obtained ranking interval range may be utilized to determine the performance stability of a unit. Shortest range between the best and the worst rankings of a hospital unit is indicative of that unit having the most stable performance of all compared hospital units. In this example, Hospital 10 is the most stable hospital unit and Hospitals 8 and 14 are the least stable ones.

\section{Managerial implications}

Comparative analysis has an important role in improving performance of production systems in assisting managers in optimizing their systems and in decision-making criteria. When input and output prices are available, profit efficiency scores help decision makers to analyse their system performance. The profit efficiency-based ranking intervals presented in this research would help decision makers to acquire information on their system ranking in comparison to other systems. Managers would be able to improve the performance of their systems with respect to other competing systems based on profit efficiency or availability of a set of feasible weights. Therefore, decision-making managers may choose to continue a process or seek improvements.

\section{Conclusions}

Basic DEA models are concerned with full information on input and output quantities without necessarily having any information on prices. Although profit model is the best model for evaluating the performance of units when input and output prices are available, profit mode could not discriminate among units with precision.

Relative performance of units based on their profit efficiency score is studies in this research while considering feasible weights. In this regard, upper and lower boundaries of profit ranking of a special DMU are determined in comparison with others by considering all alternative profit efficiency scores; each unit could achieve over a set of feasible weights.

A numerical example was finally used to illustrate and demonstrate the novelty of the introduced method.

It is recommended that further research concerning ranking intervals of DMUs be conducted based on profit efficiency with price uncertainty to address more practical situations.

Open Access This article is distributed under the terms of the Creative Commons Attribution 4.0 International License (http://crea tivecommons.org/licenses/by/4.0/), which permits unrestricted use, distribution, and reproduction in any medium, provided you give appropriate credit to the original author(s) and the source, provide a link to the Creative Commons license, and indicate if changes were made. 


\section{Appendix}

Proof of Theorem 1 Assume that the best ranking of $\mathrm{DMU}_{k}$ is achieved at $\boldsymbol{u} \in U, \boldsymbol{v} \in V$. Therefore, there must exist $L=\mathrm{PR}_{k<\text {, so that }}$

$\mathrm{PE}_{l}(\boldsymbol{u}, \boldsymbol{v})>\mathrm{PE}_{k}(\boldsymbol{u}, \boldsymbol{v}) \Rightarrow \beta_{l}^{*}(\boldsymbol{u}, \boldsymbol{v})<\beta_{k}^{*}(\boldsymbol{u}, \boldsymbol{v}) \quad$ for all $l \in L$

$\mathrm{PE}_{l}(\boldsymbol{u}, \boldsymbol{v}) \leq \mathrm{PE}_{k}(\boldsymbol{u}, \boldsymbol{v}) \Rightarrow \beta_{l}^{*}(\boldsymbol{u}, \boldsymbol{v}) \geq \beta_{k}^{*}(\boldsymbol{u}, \boldsymbol{v}) \quad$ for all $l \notin L$.

Let $\hat{\boldsymbol{u}}=\frac{\boldsymbol{u}}{-\boldsymbol{u}^{\prime} \boldsymbol{y}_{k}+\boldsymbol{v}^{\prime} \boldsymbol{x}_{k}}, \wedge=\frac{\boldsymbol{v}}{-\boldsymbol{u}^{\prime} \boldsymbol{y}_{k}+\boldsymbol{v}^{\prime} \boldsymbol{x}_{k}}$. Then $\hat{\boldsymbol{u}} \in U, \hat{\boldsymbol{v}} \in V$ and $-\hat{\boldsymbol{u}}^{\prime} \boldsymbol{y}_{k}+\hat{\boldsymbol{v}}^{\prime} \boldsymbol{x}_{k}=1$

Let $z_{l}=1(l \neq k)$ for $l \in L$ and $z_{l}=0(l \neq k)$ for $l \notin L$. $z_{\boldsymbol{l}},(\boldsymbol{l} \neq \boldsymbol{k})$ is the $l$ th component of $\boldsymbol{z}$.

So that for any $l \notin L$,

$$
\begin{aligned}
\beta_{l}^{*}(\boldsymbol{u}, v) \geq \beta_{k}^{*}(\boldsymbol{u}, v) & \Rightarrow 1 \leq \frac{\beta_{l}^{*}(\boldsymbol{u}, \boldsymbol{v})}{\beta_{k}^{*}(\boldsymbol{u}, \boldsymbol{v})}=\frac{\beta_{l}^{*}(\hat{\boldsymbol{u}}, \hat{\boldsymbol{v}})}{\beta_{k}^{*}(\hat{\boldsymbol{u}}, \hat{\boldsymbol{v}})}=\frac{\frac{-\hat{u}^{\prime} y_{l}+\hat{v}^{\prime} x_{l}}{\overline{\boldsymbol{p}}^{\prime} \hat{\boldsymbol{u}}_{l}-\overline{\boldsymbol{c}}^{\prime} \boldsymbol{x}_{l}}}{\frac{-\overline{\boldsymbol{u}}^{\prime} \boldsymbol{v}_{k} \boldsymbol{v}_{k}}{\overline{\boldsymbol{p}}^{\prime} \boldsymbol{y}_{k}-\overline{\boldsymbol{c}}^{\prime} \boldsymbol{x}_{k}}}=\frac{-\hat{\boldsymbol{u}}^{\prime} \boldsymbol{y}_{l}+\hat{\boldsymbol{v}}^{\prime} \boldsymbol{x}_{l}}{\overline{\boldsymbol{p}}^{\prime} \boldsymbol{y}_{l}-\overline{\boldsymbol{c}}^{\prime} \boldsymbol{x}_{l}} \\
& \Rightarrow-\hat{\boldsymbol{u}}^{\prime} \boldsymbol{y}_{l}+\hat{\boldsymbol{v}}^{\prime} \boldsymbol{x}_{l} \geq \overline{\boldsymbol{p}}^{\prime} \boldsymbol{y}_{l}-\overline{\boldsymbol{c}}^{\prime} \boldsymbol{x}_{l} \\
& \Rightarrow-\hat{\boldsymbol{u}}^{\prime} \boldsymbol{y}_{l}+\hat{\boldsymbol{v}}^{\prime} \boldsymbol{x}_{l}-\overline{\boldsymbol{p}}^{\prime} \boldsymbol{y}_{l}+\overline{\boldsymbol{c}}^{\prime} \boldsymbol{x}_{l} \geq 0 \\
& \Rightarrow \hat{\boldsymbol{u}}^{\prime} \boldsymbol{y}_{l}-\hat{\boldsymbol{v}}^{\prime} \boldsymbol{x}_{l}+\overline{\boldsymbol{p}}^{\prime} \boldsymbol{y}_{l}-\overline{\boldsymbol{c}}^{\prime} \boldsymbol{x}_{l} \leq 0 .
\end{aligned}
$$

Therefore;

$$
\begin{aligned}
& \beta_{l}^{*}(\boldsymbol{u}, \boldsymbol{v})<\beta_{k}^{*}(\boldsymbol{u}, \boldsymbol{v}) \Rightarrow 1>\frac{\beta_{l}^{*}(\boldsymbol{u}, \boldsymbol{v})}{\beta_{k}^{*}(\boldsymbol{u}, \boldsymbol{v})}=\frac{\beta_{l}^{*}(\hat{\boldsymbol{u}}, \hat{\boldsymbol{v}})}{\beta_{k}^{*}(\hat{\boldsymbol{u}}, \hat{\boldsymbol{v}})}=\frac{\frac{-\hat{\boldsymbol{u}}^{\prime} \boldsymbol{y}_{l}+\hat{\boldsymbol{v}}^{\prime} \boldsymbol{x}_{l}}{\overline{\boldsymbol{p}}^{\prime} \boldsymbol{y}_{l}-\overline{\boldsymbol{c}}_{l} \boldsymbol{x}_{l}}}{\frac{-\hat{\boldsymbol{u}}^{\prime} \boldsymbol{y}_{k}+\boldsymbol{v}^{\prime} \boldsymbol{x}_{k}}{\overline{\boldsymbol{p}}^{\prime} \boldsymbol{y}_{k}-\overline{\boldsymbol{c}}^{\prime} \boldsymbol{x}_{k}}}=\frac{-\hat{\boldsymbol{u}}^{\prime} \boldsymbol{y}_{l}+\hat{\boldsymbol{v}}^{\prime} \boldsymbol{x}_{l}}{\overline{\boldsymbol{p}}^{\prime} \boldsymbol{y}_{l}-\overline{\boldsymbol{c}}^{\prime} \boldsymbol{x}_{l}} \\
& \Rightarrow-\hat{\boldsymbol{u}}^{\prime} \boldsymbol{y}_{l}+\hat{\boldsymbol{v}}^{\prime} \boldsymbol{x}_{l}<\overline{\boldsymbol{p}}^{\prime} \boldsymbol{y}_{l}-\overline{\boldsymbol{c}}^{\prime} \boldsymbol{x}_{l} \\
& \Rightarrow-\hat{\boldsymbol{u}}^{\prime} \boldsymbol{y}_{l}+\hat{\boldsymbol{v}}^{\prime} \boldsymbol{x}_{l}-\overline{\boldsymbol{p}}^{\prime} \boldsymbol{y}_{l}+\overline{\boldsymbol{c}}^{\prime} \boldsymbol{x}_{l}<0 \\
& \Rightarrow \hat{\boldsymbol{u}}^{\prime} \boldsymbol{y}_{l}-\hat{\boldsymbol{v}}^{\prime} \boldsymbol{x}_{l}+\overline{\boldsymbol{p}}^{\prime} \boldsymbol{y}_{l}-\overline{\boldsymbol{c}}^{\prime} \boldsymbol{x}_{l}>0 \text {. }
\end{aligned}
$$

Considering that $z_{l}=1(l \neq k)$ for $l \in L \quad$ and $z_{l}=0(l \neq k)$ for $l \notin L$, and multiplying $z_{l}$ by $M_{k}$ points, then the first constraint is established. Moreover, solution to Model 6 is not larger than the best ranking of $\mathrm{DMU}_{k}$, because $1+\sum_{l \neq k} z_{l}=1+|L|=1+\left|\mathrm{PR}_{k<}\right|=\min \mathrm{pr}_{k<}$.

Conversely, assume optimal solution to Model 6 is obtained at $(\boldsymbol{u}, \boldsymbol{v}, \boldsymbol{z})$. Let $L=\left\{\left.l\right|_{z_{l}}=1,(l \neq k)\right\}$ for all $l \notin L$, then $z_{l}=0,(l \neq k)$. The first constraint of Model 6 reaches $\hat{\boldsymbol{u}}^{\prime} \boldsymbol{y}_{l}-\hat{\boldsymbol{v}}^{\prime} \boldsymbol{x}_{l} \leq-\overline{\boldsymbol{p}}^{\prime} \boldsymbol{y}_{l}+\overline{\boldsymbol{c}}^{\prime} \boldsymbol{x}_{l}$. Therefore,

$\frac{\beta_{l}^{*}(\boldsymbol{u}, \boldsymbol{v})}{\beta_{k}^{*}(\boldsymbol{u}, \boldsymbol{v})}=\frac{-\hat{\boldsymbol{u}}^{\prime} \boldsymbol{y}_{l}+\hat{\boldsymbol{v}}^{\prime} \boldsymbol{x}_{l}}{\overline{\boldsymbol{p}}^{\prime} \boldsymbol{y}_{l}-\overline{\boldsymbol{c}}^{\prime} \boldsymbol{x}_{l}} \geq 1 \Rightarrow \frac{\mathrm{PE}_{l}(\boldsymbol{u}, \boldsymbol{v})}{\mathrm{PE}_{k}(\boldsymbol{u}, \boldsymbol{v})} \leq 1$

is held because of the second constraint of Model 6 and the expressions of $\overline{\boldsymbol{p}}$ and $\overline{\boldsymbol{c}}$. For $l \in L$, the $\hat{\boldsymbol{u}}^{\prime} \boldsymbol{y}_{l}-\hat{\boldsymbol{v}}^{\prime} \boldsymbol{x}_{l} \leq-$ $\overline{\boldsymbol{p}}^{\prime} \boldsymbol{y}_{l}+\overline{\boldsymbol{c}}^{\prime} \boldsymbol{x}_{l}$ does not held, because setting $z_{l}=0(l \neq k)$ instead of $z_{l}=1(l \neq k)$ causes the first constraint to remain satisfied, but the objective function decreases and violates the optimality of $z$. Thus, $P R_{k<}=\mathrm{L} \quad$ and $\min \mathrm{pr}_{k<} \leq 1+\left|\mathrm{PR}_{k<}\right|=1+|L|=1+\sum_{l \neq k} z_{l}$.

Proof of Theorem 2 Proof of this theorem is similar to Theorem 1.

\section{References}

1. Charnes, A., Cooper, W.W., Rhodes, E.: Measuring the efficiency of decision making units. Eur. J. Oper. Res. 2, 429-444 (1978)

2. Färe, R., Grosskopf, S., Lovell, C.A.K.: Measurement of efficiency of production. Kluwer-Nijhoff Publishing Co., Inc, Boston (1985)

3. Färe, R., Grosskopf, S., Lovell, C.A.K.: Production frontiers. Cambridge University Press, Cambridge (1994)

4. Färe, R., Grosskopf, S.: Profit efficiency, Farrell decompositions and the Mahler inequality. Econ. Lett. 57, 283-287 (1997)

5. Chambers, R.G., Färe, R.: Additive decomposition of profit efficiency. Econ. Lett. 84, 329-334 (2004)

6. Briec, W., Comes, Ch., Kerstens, K.: Temporal technical and profit efficiency measurement: definitions, duality and aggregation results. Int. J. Prod. Econ. 103, 48-63 (2006)

7. Ruiz, J.L., Sirvent, I.: Measuring scale effects in the allocative profit efficiency. Socio. Econ. Plan. Sci. 46, 242-246 (2012)

8. Sexton, T.R., Silkman, R.H., Hogan, A.J.: Data envelopment analysis: critique and extensions. In: Silkman, R.H. (ed.) Measuring efficiency: an assessment of data envelopment analysis, pp. 73-105. Jossey-Bass, San Francisco, CA (1986)

9. Doyle, J.R., Green, R.: Efficiency and cross-efficiency in data envelopment analysis: derivatives, meanings and uses. J. Oper. Res. Soc. 45, 567-578 (1994)

10. Rodder, W., Reucher, E.: A consensual peer-based DEA-model with optimized cross-efficiencies-input allocation instead of radial reduction. Eur. J. Oper. Res. 212, 148-154 (2011)

11. Ruiz, J.L.: Cross-efficiency evaluation with directional distance functions. Eur. J. Oper. Res. 228, 181-189 (2013)

12. Yang, G.L., Yang, J.B., Liu, W.B., Li, X.X.: Cross-efficiency aggregation in DEA models using the evidential-reasoning approach. Eur. J. Oper. Res. 231, 393-404 (2013)

13. Andersen, P., Petersen, N.C.: A procedure for ranking efficient units in data envelopment analysis. Manage. Sci. 39, 1261-1264 (1993)

14. Sueyoshi, T.: Data envelopment analysis non-parametric ranking test and index measurement: slack-adjusted DEA and an application to Japanese agriculture cooperatives. Omega. 27, 315-326 (1999)

15. Seiford, L.M., Zhu, J.: Infeasibility of super-efficiency data envelopment analysis models. INFOR. 37, 174-187 (1999)

16. Chen, Y.: Ranking efficient units in DEA. Omega. 32, 213-219 (2004)

17. Lee, HSh, Chu, ChW, Zhu, J.: Super-efficiency DEA in the presence of infeasibility. Eur. J. Oper. Res. 212, 141-147 (2011)

18. Lee, HSh, Zhu, J.: Super-efficiency infeasibility and zero data in DEA. Eur. J. Oper. Res. 216, 429-433 (2012)

19. Salo, A., Punkka, A.: Ranking intervals and dominance relations for ratio-based efficiency analysis. Manage. Sci. 57, 200-214 (2011)

20. Cooper, W., Seiford, L.M., Tone, K.: Data envelopment analysis, a comprehensive text with models, applications, references and DEA-solver software. Kluwer Academic Publishers, Boston (2007)

21. Charnes, A., Cooper, W.W., Wei, Q.L., Huang, Z.M.: Cone ratio data envelopment analysis and multi-objective programming. Int. J. Syst. Sci. 20, 1099-1118 (1989)

22. Thompson, R.G., Singleton Jr., F.D., Thrall, R.M., Smith, B.A.: Comparative site evaluations for locating a high-energy physics lab in Texas. Interfaces. 16, 35-49 (1986) 